\title{
Effectiveness of the computerization of a YALE insulin infusion modified protocol in reducing the nursing workload in an Internal Medicine Department
}

\author{
Giampietro Beltramello, ${ }^{1}$ Carmelo Sgarlata, ${ }^{2}$ Marco Rollone, ${ }^{3}$ Luigi Magnani ${ }^{2}$ \\ ${ }^{1}$ Azienda Sanitaria ULSS3, Hospital of Bassano del Grappa (VI); ${ }^{2}$ Azienda Socio-Sanitaria Territoriale della Provincia di \\ Pavia, Department of Internal Medicine, Civil Hospital of Voghera, Voghera (PV); ${ }^{3}$ Azienda di Servizi alla Persona di Pavia, \\ IDR Santa Margherita, Pavia, Italy
}

\begin{abstract}
Hyperglycemia is a negative prognostic factor in both critical and non-critical patients. Many critically ill patients admitted to internal medicine wards present hyperglycemia and may benefit from an IV insulin therapy. The Yale Insulin Infusion Protocol (YIIP) is effective and safe in achieving the desired glucose range. However, to be used properly and safely, it requires a considerable availability of nursing resources. This probably represents the main limiting factor in using these protocols outside intensive care units. This study was performed to assess the effectiveness of a computer application which reduces the nursing workload related to managing a nurse implemented modified YIIP in our internal medicine department. Our experience showed that computerizing the YIIP algorithm is safe and effective. It leads on average to a net 30-min nursing time saving per day per patient.
\end{abstract}

\section{Introduction}

The management of hyperglycemia in critically ill patients has generated growing interest amongst the international scientific community in the last fifteen years. This is demonstrated by the increasing number of studies, published on this subject, sometimes with controversial results. ${ }^{1}$ It is unanimously recognized that hyperglycemia has a negative impact on mortality and on clinical outcomes in critically ill patients. There is also a

Correspondence: Giampietro Beltramello, Azienda Sanitaria ULSS3, Ospedale di Bassano del Grappa, Bassano del Grappa (VI), Italy.

E-mail: beltra.piero@gmail.com

Key words: Hyperglycemia; critically ill; YALE insulin infusion protocol; computerization, computer application; nursing workload.

Conflict of interest: the authors declare no potential conflict of interest.

Received for publication: 6 January 2018.

Revision received: 2 April 2018.

Accepted for publication: 17 April 2018.

This work is licensed under a Creative Commons Attribution NonCommercial 4.0 License (CC BY-NC 4.0).

CCopyright G. Beltramello et al., 2018

Licensee PAGEPress, Italy

Italian Journal of Medicine 2018; 12:131-136

doi:10.4081/itjm.2018.977 general consensus on the use of validated intravenous insulin infusion protocols as the most appropriate treatment for hyperglycemia. ${ }^{2-4}$ However, there is an intense debate on a number of issues. These include the definition of the correct blood glucose targets that can be achieved safely and with a reasonable use of resources. ${ }^{5}$ The choice of protocol is a complex decision that cannot be separated from a careful analysis of the specific clinical settings in which the algorithm will be implemented. The use of these protocols is not considered safe and it is not recommended in settings in which nursing support is not appropriate to accurately perform certain tasks. These include the necessary frequent blood glucose measurements and the related calculations and corrections of the insulin infusion rate (IIR). This is particularly important outside intensive care units (ICU) where fewer nursing resources are available. Also, outside ICUs the increase in nursing workload caused by the implementation of an insulin infusion protocol often limits its effective and safe utilization. In this respect, the automation of blood glucose detection systems and the use of subcutaneous devices for continuous glucose monitoring may bring great benefits. ${ }^{6,7}$ Furthermore, several studies conducted in the critical care area have shown that the computerization of the protocols brings significant advantages. It reduces errors in insulin administration and hypoglycemic episodes. At the same time, it reduces nursing workload, thereby improving efficacy and safety. ${ }^{8-11}$ To our knowledge there is no evidence of these improvements in an internal medicine setting. This, 
in spite of the lack of appropriate nursing resources being the main limitation on the use of intravenous insulin protocols, which are therefore underused. This study was performed to assess and quantify the effectiveness in using a computer application developed to automate the calculations required for the management of a nurse-implemented modified Yale Insulin Infusion Protocol (YIIP). The aim of the assessment is to reduce the related nursing workload in an internal medicine department.

\section{Materials and Methods}

The study enrolled 78 consecutive diabetic patients who were admitted to the internal medicine ward of San Bassiano Hospital (Bassano del Grappa, Vicenza, Italy) from the emergency department of the same hospital between December 2013 and December 2015. All patients met both the following inclusion criteria: i) blood glucose of at least 200 $\mathrm{mg} / \mathrm{dL}$ evaluated on blood sample collected upon admission; ii) presence of an acute or chronic exacerbated illness. Patients with a known history of terminal illness (either oncologic or not) were excluded from the study $(\mathrm{n}=5)$. Personal data (age, gender), admission diagnoses and entry blood glucose levels from venous blood samples were collected for each patient. A nurse-implemented modified YIIP, already in use at our department, was applied to all enrolled patients with a glycemic target of $>140$ and $<180 \mathrm{mg} / \mathrm{dL}$. The same protocol was used for all patients but was applied in two different ways, thereby creating two groups. The first group comprised the first 38 consecutive patients enrolled. The conventional method in use in our department was applied to this group. This involved calculating manually on paper the variations of insulin infusion rate. The second group consisted of the subsequent 35 patients enrolled. For this group all the calculations required for managing insulin infusion rates were performed automatically through a computer application. For this purpose we developed a Microsoft Windows application with a simple and intuitive interface (Figure 1). The application automatically calculates the variations of the insulin infusion rate according to the parameters defined by the modified YIIP. This software was installed on a computer used by ward nurses. All nurses involved in the study were trained for $30 \mathrm{~min}$ on how to use the application. For each patient blood glucose levels were detected and registered at specified intervals as provided by the algorithm. The IIR was corrected on the basis of three main parameters: the measured value of capillary blood glucose; the blood glucose value at the previous detection; and the IIR itself.

\section{Nursing workload quantification}

The nursing workload required for applying the protocol was quantified through the following method. We first measured the time it took 10 skilled nurses to perform a capillary blood glucose determination. We also measured the time needed to determine the insulin infusion rate using both the conventional method (i.e. performing all calculations on paper) and the computer application. We then calculated average times for both methods. Finally, we calculated the average number of daily capillary blood glucose readings per day for each patient during the application of the protocol. The average total nursing time required per day for the application of the protocol for each patient was then calculated by: i) summing the average time required to determine the capillary blood glucose with the average time required to calculate the infusion rate; and then ii) multiplying those values by the average number of daily capillary glucose readings per day for each patient. In addition, we asked all the nurses involved in the study to complete a short anonymous questionnaire with the following questions: i) Do you believe that the computerization of the protocol reduced your workload?; ii) If you could choose, would you rather continue to apply the protocol by using the conventional method or the computer application?. It was not among the objectives of this study to evaluate the effectiveness of the algorithm. Nevertheless, we calculated for both groups the percentage of patients which reached the glycemic target (i.e. blood glucose between $140-180 \mathrm{mg} / \mathrm{dL}$ ) after $12 \mathrm{~h}$ from the start of insulin infusion. We also recorded all hypoglycemic episodes (blood glucose $<60 \mathrm{mg} / \mathrm{dL}$ ).

\section{Statistical analysis}

For the statistical analysis we used the software MEDCALC 12.1.4.0 version for Windows. We expressed continuous variables as mean values \pm standard deviation, and categorical variables as percentages.

\section{Results}

The study enrolled 78 patients who fulfilled the inclusion criteria. Of these, 5 were excluded. Therefore the study population included 73 patients (42 males, 31 females) with a mean ( \pm standard deviation) age of $71.2( \pm 6.3)$ years. Admission diagnoses were the following: heart failure in $24 / 73$ patients $(32.87 \%)$; pneumonia in $12 / 73(16.43 \%)$; acute coronary syndromes in 6/73 (8.21\%); sepsis in 14/73 (19.17\%); obstructive disventilatory syndrome (chronic obstructive pulmonary disease, asthma, bronchitis) in $12 / 73(16.43 \%)$; and pulmonary 
embolism in 5/73 (6.84\%). Table 1 shows a summary of the characteristics of study population.

In the first group of patients, for whom the insulin protocol was managed according to the conventional method, the average blood glucose value before the infusion was $364.8 \mathrm{mg} / \mathrm{dL}$. Twelve and $24 \mathrm{~h}$ after the start of the protocol blood glucose levels were 121 $\mathrm{mg} / \mathrm{dL}$ and $131.2 \mathrm{mg} / \mathrm{dL}$, respectively. Stable mean blood glucose values were found up to 36 hours after the start of the protocol. In the second group of patients, for whom the insulin protocol was managed through the computer application, the average blood glucose value before the beginning of the infusion was $312.4 \mathrm{mg} / \mathrm{dL}$. Twelve and $24 \mathrm{~h}$ after the start of the protocol blood glucose levels were $154.5 \mathrm{mg} / \mathrm{dL}$ and $122.5 \mathrm{mg} / \mathrm{dL}$, respectively. Also for this group stable mean blood glucose values were found up to $36 \mathrm{~h}$ after the start of the protocol. The average time it took nursing staff to determine the capillary blood glucose was $2 \mathrm{~min}$. The average time to calculate the variations of the IIR was 3 min with the conventional paper- based calculation method and only 1 min with the computer application. On the whole enrolled population we performed on average 15 capillary blood glucose determinations per patient in $24 \mathrm{~h}$. The mean total nursing time to manage the protocol in the $24 \mathrm{~h}$ was $75 \mathrm{~min}$ for the first group and $45 \mathrm{~min}$ for the

Table 1. Characteristics of study population.

\begin{tabular}{lc}
\hline Total population & $\mathrm{n}=73$ \\
\hline Sex (male) & $42 / 73(57.53 \%)$ \\
\hline Age (median IQR) years & $71.2 \pm 6.3$ years \\
\hline Admission diagnosis & \\
Heart failure & $32.87 \%(\mathrm{n}=24)$ \\
Pneumonia & $16.43 \%(\mathrm{n}=12)$ \\
Acute coronary syndrome & $8.21 \%(\mathrm{n}=6)$ \\
Sepsis & $19.17 \%(\mathrm{n}=14)$ \\
Obstructive disventilatory syndrome & $16.43 \%(\mathrm{n}=12)$ \\
Pulmonary embolism & $6.84 \%(\mathrm{n}=5)$ \\
\hline
\end{tabular}

\section{Variazione della velocità di infusione}

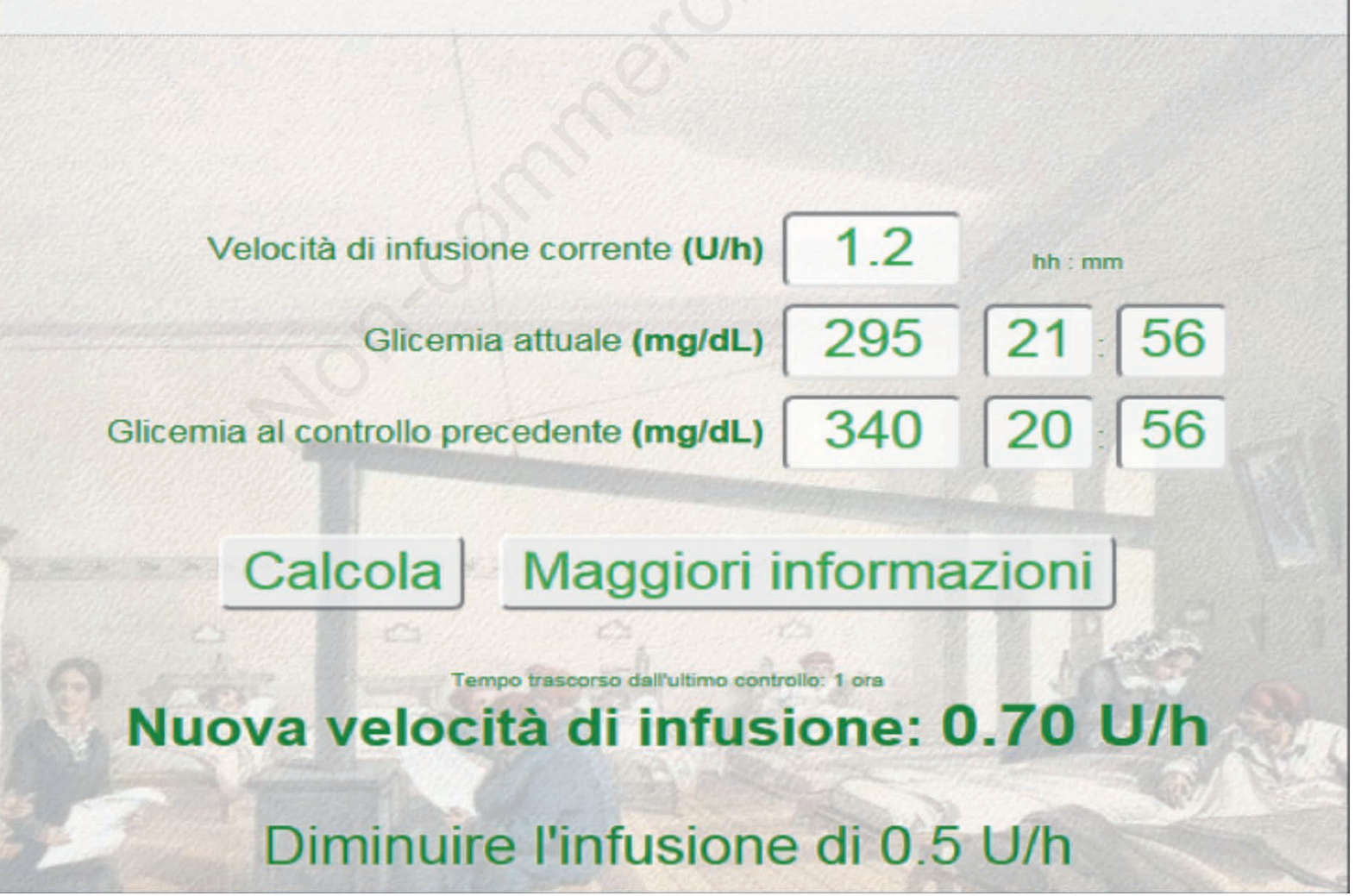

Figure 1. Graphic interface of the computer application used for the calculation of insulin infusion rate variations [the software is in Italian language]. The compilation of three fields Actual infusion rate (Velocità di infusione corrente), Actual blood glucose (Glicemia attuale), and Blood glucose at the previous control (Glicemia al controllo precedente) provides an instant calculation of the new infusion rate. 
second group. All 10 nurses involved in the study replied to the questionnaire. All of them declared that the use of the computer application reduced their workload and that they would prefer to apply the protocol by using the computer system if given the choice. The percentage of patients reaching the glycemic target after $12 \mathrm{~h}$ was $65.78 \%(\mathrm{n}=25)$ in the first group and $77.14 \%(\mathrm{n}=27)$ in the second group. Seven hypoglycemic episodes occurred in the first group while none occurred in the second group (Figure 2). Data are not statistically significant.

\section{Discussion and Conclusions}

This study shows that the use of a computer application can give an important contribution in reducing the nursing workload related to the application of an intravenous insulin protocol in an Internal Medicine Department. The high absorption of nursing resources is the major factor limiting the use of such protocols outside the intensive care areas. This is because the implementation of these algorithms is not considered safe. Nor is it recommended where nursing support is not sufficient to accurately perform all the blood glucose readings and all the therapeutic corrections (infusion rate variations) provided by the protocols. ${ }^{12}$ This is probably why these algorithms are extremely underused in internal medicine. This is the case even though it is well known that the presence and severity of hyperglycemia represents an independent adverse prognostic factor in critically ill patients. It also correlates closely with the increase in mortality. ${ }^{13}$ Indeed, there is extensive evidence showing the relationship between hyperglycemia and decreased survival in many medical and surgical acute diseases. ${ }^{14-16}$ For some pathological conditions such as heart failure and acute myocardial infarction, it has been also shown that there is a significant correlation with hyperglycemia during hospitalization both with the increase of hospital mortality and with long-term mortality. ${ }^{15,17}$ For these reasons there is a general consensus on the need to treat hyperglycemia in critically ill patients. All major National and International guidelines ${ }^{3,18}$ agree on recommending the use of continuous intravenous insulin infusion in case of persistent hyperglycemia $(>180 \mathrm{mg} / \mathrm{dL})$ and on advising the maintenance of blood glucose values between 140 and $180 \mathrm{mg} / \mathrm{dL}$ in most cases. An additional difficulty in implementing these protocols in internal medicine departments is represented by the lack of universally shared and objective criteria to define critical illnesses. This is because the numerous scores often used in ICU are not applicable in our departments, since they are calibrated on a very high threshold of severity. In this study we considered it reasonable to classify patients as critically ill on the basis of the overall clinical evaluation performed by the ward physician at the time of patient admission. There are many validated algorithms to manage the insulin infusion therapy ${ }^{19}$ and no randomized clinical trials were carried out to compare the different protocols in terms of morbidity and mortality. ${ }^{20}$ However, an ideal IIT protocol should allow to reach quickly and safely the glucose target value (low glycemic variability, low risk of hypoglycemia). It should also be easy to implement with a reasonable resource expenditure. The Yale Protocol (and its variants) is one of the most widely used algorithms in medical area. It is a dynamic algorithm: to calculate

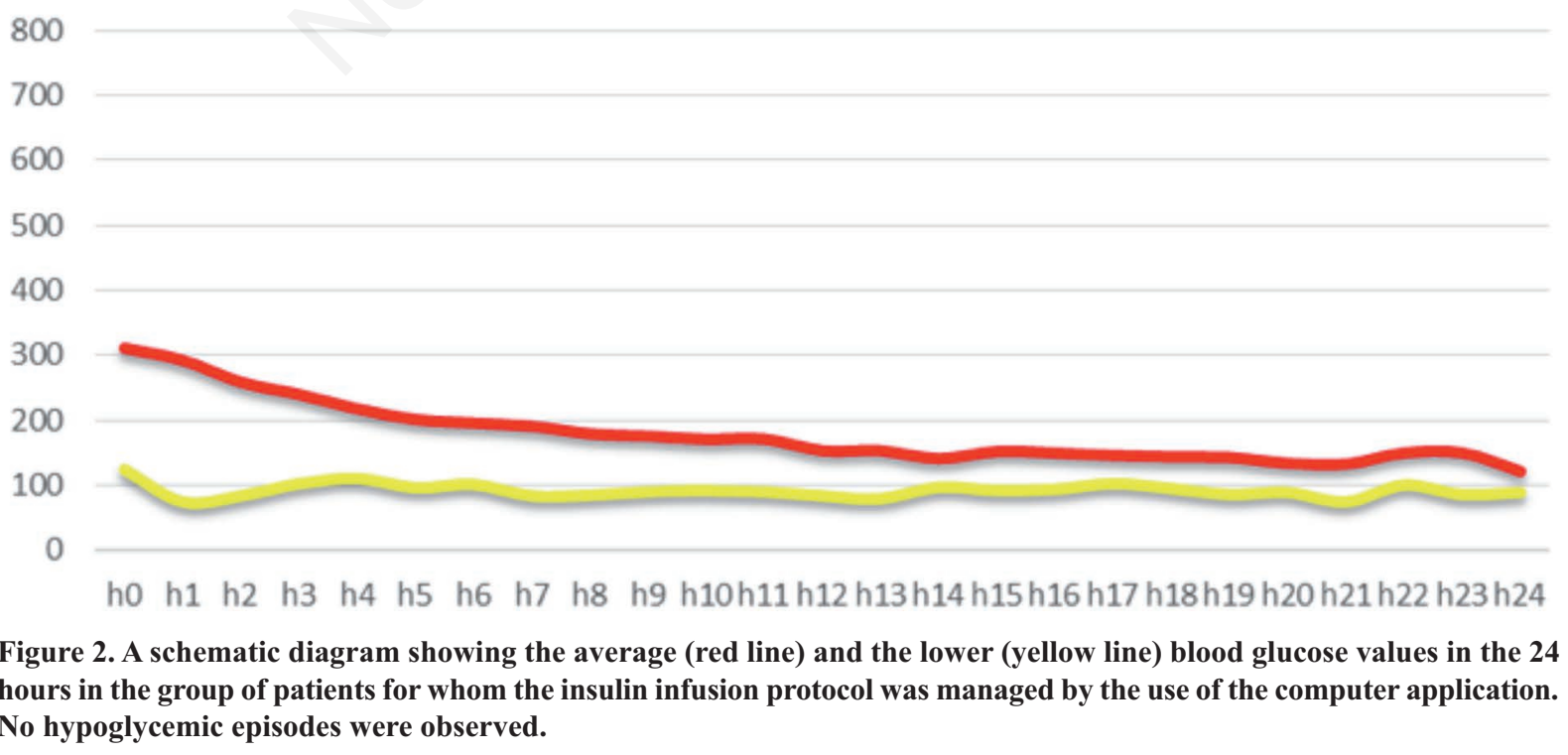


insulin dosing it considers not only the absolute value of glycemia but also the glycemic trend, i.e. the direction and the speed of the blood glucose values changes. These characteristics allow a very fine adjustment of the insulin dose by reducing the risk of hypoglycemia and glucose variability. However, the proper and safe application of the protocol requires an adequate availability of nursing resources for the execution of all the necessary blood glucose readings and for calculating the variations of the IIR. The time required for blood glucose reading cannot be reduced without the use of complex automatic detection systems, whose introduction is currently not feasible in internal medicine wards. Our study, indeed, showed that the average total daily nursing time required for the application of the protocol can be greatly reduced by computerizing the calculation processes. Furthermore, the computerization of the method eliminated the need to manually perform all the algorithm calculations needed to determine the changes of the IIR; our study has also shown that both simplifying and optimizing the algorithm significantly reduce the time required for such operations. This led to a considerable saving of nursing resources equal on average - to $30 \mathrm{~min}$ of nurse work per patient per day. This result was obtained without hampering the safety and the effectiveness of the protocol. This is evidenced by the total absence of hypoglycemic episodes in the group of patients for which the insulin infusion protocol was managed using the computer application. It is also evidenced by the comparable proportion of patients which reached the glycemic target in the two groups. Our data also suggest that the computerization of the protocol could lead to a reduction of the hypoglycemic episodes. At the same time, the smallness of our sample did not allow us to obtain a statistically significant safety comparison between the two groups. A further advantage given by the use of the computer application is that it allows not fully skilled nurses to use the protocol. This is because they no longer need to learn how to perform all the calculations; they just need a short training period to learn how to use the computer application. This ensures continuity in the use of the protocol even in the presence of a high nursing turnover. Finally, the results obtained from the questionnaire show that the nursing staff prefers the use of this application over the conventional method, with obvious positive implications for compliance. The major limit of this study is the small number of patients enrolled which did not allow a statistically significant comparison in terms of efficacy and safety. However this was not the goal of our work. Instead, we showed how the computerization of a nurse-implemented modified YIIP can speed up and simplify its application. This widens the possibilities of its use in an internal medicine department by reducing the absorption of nursing resources.

\section{References}

1. Van Van den Berghe G, Wouters P, Weekers F, et al. Intensive insulin therapy in critically ill patients. N Engl J Med 2001;345:1359-67.

2. Beltramello G, et al. Trialogue: managing hyperglycaemia in internal medicine. Instructions for use. Ital J Med 2012;6:123-32.

3. Associazione Medici Diabetologi (AMD). Società Italiana di Diabetologia (SID). Standard italiani per la cura del diabete mellito; 2014, pp 5-9.

4. American Diabetes Association. (13) Diabetes care in the hospital, nursing home, and skilled nursing facility. Diabetes Care 2015;38:S80-5.

5. Jacobi J, Bircher N, Krinsley J, et al. Guidelines for the use of an insulin infusion for the management of hyperglycemia in critically ill patients. Crit Care Med 2012;40:3251-76.

6. Holzinger U, Warszawska J, Kitzberger R, et al. Realtime continuous glucose monitoring in critically ill patients. Diabetes Care 2010;33:467.

7. Schaupp L. Prediction of glucose concentration in postcardiothoracic surgery patients using continuous glucose monitoring. diabetes technol. Therapy 2011;13:127-34.

8. Bouw JW. A retrospective cohort study of a nurse-driven computerized insulin infusion program versus a paperbased protocol in critically ill patients. Diabetes Technol Ther 2012;14:125-30.

9. Marvin MR, Inzucchi SE, Besterman BJ. Computerization of the Yale insulin infusion protocol and potential insights into causes of hypoglycemia with intravenous insulin. Diabetes Technol Ther 2013;15:246-52.

10. Vogelzang M, Loef BG, Regtien JG, et al. Computerassisted glucose control in critically ill patients. Intensive Care Med 2008;34:1421-7.

11. Davidson PC, Steed DR, Bode BW, et al. Use of a computerized intravenous insulin algorithm within a nurse-directed protocol for patients undergoing cardiovascular surgery. J Diabetes Sci Technol 2008;2: 369-75.

12. Moghissi ES, Korytkowski MT, DiNardo M, et al. American Association of Clinical Endocrinologists and American Diabetes Association consensus statement on inpatient glycemic control. Diabetes Care 2009;32: 1119-31.

13. Krinsley JS. Association between hyperglycemia and increased hospital mortality in a heterogeneous population of critically ill patients. Mayo Clin Proc 2003;78:1471-8.

14. Falciglia M, Freyberg RW, Almenoff PL, et al. Hyperglycemia-related mortality in critically ill patients varies with admission diagnosis. Crit Care 2010;37: 3001-9.

15. Deckers JW, van Domburg RT, Akkerhuis M, Nauta ST. Relation of admission glucose levels, short- and longterm (20-year) mortality after acute myocardial infarction. Am J Cardiol 2013;112:1306-10. 
16. Mebazaa A, Gayat E, Lassus J, et al. Association between elevated blood glucose and outcome in acute heart failure: results from an international observational cohort. J Am Coll Cardiol 2013;61:820-9.

17. Ukena C, Dobre D, Mahfoud F, et al. Hypo- and hyperglycemia predict outcome in patients with left ventricular dysfunction after acute myocardial infarction: data from EPHESUS. J Card Fail 2012;18: 439-45.
18. American Diabetes Association. 13. Diabetes Care in the Hospital: Table 13.1. Diabetes Care 2016;39:S99S104.

19. Boutin J-M, Gauthier L. Insulin infusion therapy in critically ill patients. Can J Diabetes 2014;38:144-50.

20. Krikorian A, Ismail-Beigi F, Moghissi ES. Comparisons of different insulin infusion protocols: a review of recent literature. Curr Opin Clin Nutr Metab Care 2010;13: 198-204. 